\title{
Index to Volume 131
}

\section{Compiled by William Halliday}

Abies balsamea, 151

Abundance, 128

Acoustic, Monitoring, 10

Acoustic location system, 10

Agelaius phoeniceus, 312

Airport, Vancouver International, 26

Alces americanus, 215

Alston, J.M., J.E. Millard, J.A. Rick, B.W. Husby, L.A. Mundy. Observations of Notable Parental Behaviours of Northern Spotted Owls (Strix occiden-

Alvar, 75 talis caurina), 225-227

Amphibian decline, 115

Analysis, Meta, 1

Anderson, E.M., 26

Ant, European Fire, 347

Arctic, 23

Ardea Herodias, 369

Aspen, Large-toothed, 128

Balaena mysticetus, 270

Barbe, M., L. Dubois, J. Faubert, M. Lavoie, Y. Bergeron, N.J. Fenton. Range Extensions of 35 Bryophyte Species in the Black Spruce-Feather Moss Forest of Western Quebec, Canada, 258-269

Baril, L.M., D.B. Haines, L.E. Walker, D.W. Smith. Autumn Raptor Migration in Yellowstone National Park, 303-311

Baselines, Ecological, 350

Basquill, S., 164

Bay, Constance, 128

Beachcast, 270

Bear, Black, 225

Beaver, American, 215

Beazley, K.F., 32

Behaviour, 23

Defensive, 235

Extinguished learning, 23 Foraging, 125

Bell, K.A.H., 37

Bergeron, Y., 258

Bety, J., 203

Bioherms, 46

Bird(s), 303

\section{Boreal, 55}

Colonial Nesting, 369

Grassland, 170

BioBlitz, 386

Bison, 221

Bison bison, 221
Blackbird, Red-winged, 312

Blaney, S., 164

Blouin-Demers, G., 228

Boreal, 151

Boudreau, M.R., 344

Bradbeer, D., 26

Breeding, 69, 338, 344

Brigham, R.M., 125

British Columbia

Bag Harbour, 350

Brandywine Falls, 144

Haida Gwaii, 350

Howe Sound, 46

Okanagan Valley, 133

Saanich, 37

Similkameen Valley, 133

Vancouver, 26

Vancouver Island, 120

Brodo, I., 111

Brownsnake

Dekay's, 235

Northern, 235

Brunton, D.F., P.M. Catling. Thematic Collection: Alvars in Canada, 75-79

Bryo-geography, 258

Bryoflora, 258

Bryophyta, 246, 258

Bucephala albeola, 338

Bufflehead, 338

Buse, A.J., M.A. Douglas, T.N. Giguere, B.W. Robinson. Adult Snapping Turtle (Chelydra serpentina) Feeding on Goldeneye Embryos of Pumpkinseed (Lepomis gibbosus) in Defended Nests, 254-257

Butterfly, 63

Calidris pusilla, 203

Callaghan, C. 301

Calliphoridae, 350

Cambarus bartonii bartonii, 335

Canada, 75, 179

Caners, R.T. Fabronia ciliaris, a Moss New to Canada from Southeastern Manitoba, 246-251

Canids, 215

Canis

lupus, 23, 32, 215, 252

lycaon, 32

Cape

Graham Moore, 69

Hay, 69 
Caribou, Woodland, 215

Castor canadensis, 215

Catling, P.M., 63, 75

Catling, P.M., B. Kostiuk. Reduced Diversity and Relative Abundance of Terrestrial Snails in a Red Pine Plantation Compared with a Surrounding Red Oak - Large-toothed Aspen Woods, 128132

Catling, P.M., B. Kostiuk, J. Heron, R. Jimenez, M. Chapman, S. Gamiet, V. Sterenberg. Highlights from the Northwest Territories BioBlitzes, 386396

Census, Population, 69

Chapman, C.J., M.J. Oldham. Reversed Clover, Trifolium resupinatum $\mathrm{L}$. (Fabaceae), Confirmed in Canada, 328-330

Chapman, M., 386

Change,

Climate, 55

Forest, 151

Chelydra serpentina, 254

Chickadee, Black-capped, 10

Chordeiles minor, 125

Cipriani, J., 111

Citizen science, 46, 115

Clayton, L., G. Dennison. Inexpensive Video Dropcamera for Surveying Sensitive Benthic Habitats: Applications from Glass Sponge (Hexactinellida) Reefs in Howe Sound, British Columbia, 46-54

Climate change, 55

Clover

Persian, 328

Reversed, 328

Colony, 369

Colour, 335

Conservation, 164, 203, 397

Cormorant, Double-crested, 347

Corvus, 350

Cottontail, Nuttall's, 133

Count, Point, 10

Crayfish, Appalachian Brook, 335

Cypripedium reginae, 63

Cypseloides niger, 144

Cyr, M.-A., 69

Darbyshire, S., S. Blaney, S. Basquill. The First Record for Altai Fescue, Festuca altaica (Poaceae), in Nova Scotia, from an Eastern Alpine Site on Cape Breton Island, 164-169

Dealfish, 325

Deer, White-tailed, 151

Defense, Nest, 225

Dennison, G., 46

Density

Ant Nest, 347

Population, 170, 228
Dermochelys coriacea, 120

Diet, 19, 26, 215, 225, 254

Dispersal, 221

Fungi, 238

Distribution, 179, 246

Historical, 32

Negative binomial, 63

Disturbance, Human, 215

Diversity, 128

Dixon-MacCallum, G.P., K.A.H. Bell, P.T. Gregory. Edge-habitat Use by Northwestern Gartersnakes (Thamnophis ordinoides) in Saanich, British Columbia, 37-45

Dolphin, Pacific White-sided, 120

Douglas, M.A., 254

Drop-camera, 46

Dubois, L., 258

Eagle, Bald, 369

Ecology, 203

Editorial, 397

Editors' Report for Volume 130 (2016), 301-302

Ellis-Felege, S.N., C.G. Skaggs, G.A. Knutsen. Increased Bufflehead (Bucephala albeola) Breeding Activity in Minnesota, 338-343

Embryos, Fish, 254

Eozapus, 141

Esox niger, 331

Estuary, St. Lawrence River, 203

Evidence, Anecdotal, 32

Expansion, 338

Fabaceae, 328

Fabronia

ciliaris, 246

pusilla, 246

Fabroniaceae, 246

Falco columbarius, 344

Falls, Brandywine, 144

Faubert, J., 258

Fenton, 258

Fescue, Altai, 164

Festuca altaica, 164

Fir, Balsam, 151

Fitzsimmons, L.P., 10

Flight ability, 125

Floristics, 164

Flow, Energy, 350

Flyway, Rocky Mountain, 303

Food Web, 350

Foote, J.R., L.P. Fitzsimmons, L.M. Lobert, L.M. Ratcliffe, D.J. Mennill. A Population-level Analysis of Morning Song: Exploring the Implications for Point Counts, 10-18

Forest

Ancient, 350

Boreal, 151, 258 
Found, R., A.A.D. McLaren, A.R. Rodgers, B.R. Patterson. Diet of Grey Wolves (Canis lupus) During Calving in a Moose-Caribou System in Northern Ontario, 215-220

Fox, Red, 19

Fragmentation, 133

Fraser, G.S., 347

Frey, J.K. A Review and the Conservation Implications of Aquatic Behaviour and Drowning in Jumping Mice (Dipodidae: Zapodinae), 141-143

Frog, Wood, 386

Fungi

Cave, 238

Cold-tolerant, 238

Gable, T.D., S.K. Windels, I.C. Rautio. River Otter (Lontra canadensis) Killed by Wolves (Canis lupus) during Winter in Northern Minnesota, 252-253

Gamiet, S., 386

Gartersnake

Common, 228

Northwestern, 37

Gaston, A.J., M.-A. Cyr, K. O’Donovan. A First Count of Thick-billed Murres (Uria lomvia) and Blacklegged Kittiwakes (Rissa tridactyla) Breeding on Bylot Island, 69-74

Giguere, T.N., 254

Grassland, Fescue, 170

Grebe

Horned, 317

Red-necked, 317

Gregory, P.T., 37

Guano, 347

Gulf, Amundsen, 270

Gupta, A., K. Rudmik, G.S. Fraser. Evidence for a Negative Effect of Double-crested Cormorants (Phalacrocorax auritus) on Invasive European Fire Ants (Myrmica rubra), 347-349

Gynandromorphy, Bicoloured, 335

Habitats, 228

Sensitive benthic, 46

Haines, D.B., 303

Haliaeetus leucocephalus, 369

Hall, P., 270

Hall, P.W., P.M. Catling, P.L. Mosquin, T. Mosquin. European Skipper Butterfly (Thymelicus lineola) Associated with Reduced Seed Development of Showy Lady's-slipper Orchid (Cypripedium reginae), 63-68

Halliday, W.D. Publication Trends in The Canadian Field-Naturalist, 1980-2015, 1-9

Hammell, G. Changes to the Population Status of Horned Grebes (Podiceps auritus) and Red- necked Grebes (Podiceps grisegena) in Southwestern Manitoba, Canada, 317-324

Hanrahan, C., 111

Hartzell, S.M. A Bilaterally Partitioned Colour Variant of an Appalachian Brook Crayfish (Cambarus bartonii bartonii) from Eastern Pennsylvania, 335-337

Harwood, L.A., E.V. Lea, S.A. Raverty, P.A. Hall, E. Linn, L. Postma, O. Nielsen. Observations of Beachcast Bowhead Whales (Balaena mysticetus) in the Southeastern Beaufort Sea and Amundsen Gulf, 1987-2016, 270-279

Herbivory, 151

Herdman, E.J., K.E. Hodges. Habitat Use by Nuttall's Cottontails (Sylvilagus nuttalli nuttalli) at their Northern Range Edge (British Columbia, Canada), 133-140

Heron, Great Blue, 369

Heron, J., 386

Hexactinellida, 46

Hirundo rustica, 26

Hodges, K.E., 133

Holst, M. Marine Mammal and Sea Turtle Sightings During a Survey of the Endeavor Segment of the Juan de Fuca Ridge, British Columbia, 120-124

Husby, B.W., 225

Igl, L.D. Sora (Porzana carolina) Parasitism of Redwinged Blackbird (Agelaius phoeniceus) Nests, 312-315

Insectivore, Aerial, 26, 144

Interaction, Predator-prey, 254

Interference, Pollinator, 63

Island
Bylot, 69
Cape Breton, 164
Ellesmere, 23
Moresby, 350

Jimenez, R., 386

Jung, T.S., N.C. Larter. Observations of Long-distance Post-release Dispersal by Reintroduced Bison (Bison bison), 221-224

Kirschbaum, J., 151

Kittiwake, Black-legged, 69

Knutsen, G.A., 338

Kostiuk, B., 128, 386

Labrador, Torngat Mountains, 55

Lady's-slipper, Showy, 63

Lagenorhynchus obliquidens, 120

Lake

Ontario, 331

Simcoe, 369

Superior, 151 
Lamarre, J.-F., 203

Lamoureux, R.P., M.R. Boudreau, J.L. Seguin. New Breeding Record for Merlin (Falco columbarius) in Southwestern Yukon, 344-346

Larter, N.C., 221

Larus, 350

Lavoie, M., 258

Law, A.A., M.E. Threlfall, B.A. Tijman, E.M. Anderson, S. McCann, G. Searing, D. Bradbeer. Diet and Prey Selection of Barn Swallows (Hirundo rustica) at Vancouver International Airport, 2631

Lea, E.V., 270

Learned-response, 23

Learning, 23

LeGros, D.L. Side to Side Swaying as a Defensive Behaviour in the Dekay's Brownsnake (Storeria dekayi), 235-237

Lepage, D. Minutes of the $138^{\text {th }}$ Annual Business Meeting of the Ottawa Field-Naturalists' Club January $10,2017,96-110$

Lepitzki, D. Editorial, 397

Lepitzki, D., C. Callaghan, A. Martin. Editors' Report for Volume 130 (2016), 301-302

Lepomis gibbosus, 254

Levesque, P.G., C.A. Rock. Searching for Black Swift (Cypseloides niger) Nests in Southern British Columbia, 144-150

Linn, E., 270

Lithobates sylvaticus, 386

Liverwort, 258

Lobert, L.M., 10

Lontra canadensis, 252

Malloch, D., 238

Mallon, E., 115

Mammal, Marine, 120

Manitoba

Prairie-potholes, 317

Southeastern, 246

Marine mammal, 120

Marine protected area, Endeavour hydrothermal vent, 120

Maritime, 32

Martin, A., 301

Martin, A.E. Thematic Collection: The Canadian FieldNaturalist, Documenting Species New to Canada for Nearly a Century, 179-186

McAlpine, D.F., 238

McCann, S., 26

McGuire, L.P., R.M. Brigham. Common Nighthawks (Chordeiles minor) Can Take Off from Water, 125-127

McLachlan Hamilton, K., 111

McLaren, A.A.D., 215

Mech, D.L. Extinguishing a Learned Response in a Free-ranging Gray Wolf (Canis lupus), 23-25
Mennill, D.J., 10

Merlin, 344

Meta-analysis, 1

Migration, 303

Fall, 203

Spawning, 350

Migration timing, 203

Millard, J.E., 225

Minnesota, 151

Great Plains, 338

Marshall County, 338

Northern, 252

Voyageurs National Park, 252

Minutes of the $138^{\text {th }}$ Annual Business Meeting of the Ottawa Field-Naturalists' Club January 10, 2017, 96-110

Mirounga angustirostris, 120

Monitoring

Acoustic, 10

Browse ecological, 151

Passive acoustic, 10

Moore, D.J., 331

Moose, 215

Morrison, B.P., D.J. Moore. First Occurrence of a Juvenile Chain Pickerel (Esox niger) in Ontario Waters of Lake Ontario, 331-334

Mosquin, P.L., 63

Mosquin, T., 63

Moss, 246, 258

True, 258

Mountains, Torngat, 55

Mouse,

Deer, 238

Jumping, 141

Movement, 133, 221

Mundy, L.A., 225

Murre, Thick-billed, 69

Mycota, Cave, 238

Myrmica rubra, 347

Napaeozapus, 141

National Monument, Grand Portage, 151

National Park

Kluane, 344

Sirmilik, 69

Voyageurs, 252

Yellowstone, 303

National Wildlife Refuge

Agassiz, 338

Great Swamp, 19

Natural history, 1

Nesting, 144, 254, 338

Cavity, 338

New Brunswick, Sackville, 238

New Jersey, 19

Newfoundland, Bay of Exploits, 325

Nielsen, O., 270 
Nighthawk, Common, 125

Non-native, 347

North Dakota, Northern Prairie Wildlife Research Center, 312

Northwest Territories, 386

Nova Scotia, Cape Breton Island, 164

Novisuccinea ovalis, 128

Nunuvut

Bylot Island, 69

Ellesmere Island, 23

O’Donovan, K., 69

Oak, Northern Red, 128

Odocoileus virginianus, 151

Oldham, M.J., 328

Oncorhynchus, 350

Ontario, 115, 328

Constance Bay, 128

Kingston, 10

Lake Ontario, 331

Northern, 215

Ottawa Valley, 63

Rondeau Provincial Park, 235

Orchid, 63

Otter, River, 252

Owl, Northern Spotted, 225

Parasitism, Brood, 312

Park, Gatineau, 228

Partition, Bilateral, 335

Passive acoustic monitoring, 10

Patterson, B.R., 215

Pennsylvania, Stillwater, 335

Peromyscus maniculatus, 238

Phalacrocorax auritus, 347

Phalarope, Red-necked, 386

Phalaropus lobatus, 386

Phocoenoides dalli, 120

Physeter macrocephalus, 120

Phytogeography, 246, 258

Pickerel, Chain, 331

Pine, Red, 128

Pinus resinosa, 128

Plantation, Pine, 128

Plants, Rare, 164

Plethodon cinereus, 115

Podiceps

auritus, 317

grisegena, 317

Poecile atricapillus, 10

Pollination, 63

Population, Peripheral, 133

Status, 317

Populus grandidentata, 128

Porpoise, Dall's, 120

Porzana carolina, 312
Post-release, 221

Postma, L., 270

Predation, 215, 254

Nest, 225, 254, 369

Prey, 19, 26, 215, 254

Provinces, Maritime, 32

Provincial Park

Rondeau, 235

Whiteshell, 246

Pseudogymnoascus destructans, 238

Pumpkinseed, 254

Purchase, C.F. First Report of Dealfish, Trachipterus arcticus (Lampriformes: Trachipteridae), from Canadian Waters, 325-327

Pylypec, B. Trends in Bird Densities at a Remnant Fescue Grassland in Saskatchewan, 170-178

Quebec

Gatineau Park, 228

Northern, 258

St. Lawrence River Estuary, 203

Quercus rubra, 128

Ram-feeding, 254

Range

Breeding, 338, 344

Extension, 258, 325, 328, 331

Shifts, 55, 179, 258, 325, 328, 331

Rangifer tarandus, 215

Raptor, 303

Ratcliffe, L.M., 10

Rate, Detection, 10

Rautio, I.C., 252

Raverty, S.A., 270

Record, New, 328

Recording, Remote video, 254

Reef, Glass sponge, 46

Regression, Matched-pairs logistic, 37

Reimchen, T.E. Diverse Ecological Pathways of Salmon Nutrients Through an Intact Marine-terrestrial Interface, 350-368

Reintroduction, 221

Reproduction, 369

Reptiles, 37

Retamal Diaz, F., G. Blouin-Demers. Northern Snakes Appear Much More Abundant in Old Fields than in Forests, 228-234

Review, Literature, 1, 32, 75, 141, 179

Rick, J.A., 225

Rissa tridactyla, 69

River, North Branch Susquehanna, 335

Robinson, B.W., 254

Rock, C.A., 144

Rodgers, A.R., 215

Rosa acicularis, 386

Rose, Prickly, 386

Rudmik, K., 347 
Sagebrush-steppe, 133

Salamander, Eastern Red-backed, 115

Sanders, S., J. Kirschbaum. Short-term Change in Forest Metrics at Grand Portage National Monument, Minnesota, 151-163

Sandpiper, Semipalmated, 203

Saskatchewan, Saskatoon, 170

Saussurea angustifolia, 386

Savannah, 128

Saw-wort, Narrow-leaved, 386

Scat, 215

Science, Citizen, 46, 115

Sea

Beaufort, 270

Salish, 46

Seal, Northern Elephant, 120

Searing, G., 26

Seburn, D.C., E. Mallon. Has the Eastern Red-backed Salamander (Plethodon cinereus) Declined in Ontario?, 115-119

Seguin, J.L., 344

Selection

Habitat, 228

Prey, 26, 215

Serfass, T.L., 19

Shorebirds, 203

Shrubification, 55

Site, Staging, 203

Skaggs, C.G., 338

Skipper, European, 63

Smith, D.W., 303

Snails,

Land, 128

Terrestrial, 128

Snake, Red-bellied, 228

Soil, 347

Song, Dawn, 10

Sora, 312

Spawning, 350

Species

At risk, 317

Endangered, 141

Introduction, 179

Invasive, 347

Sphagna, 258

Sponge, Glass, 46

Sterenberg, V., 386

Storeria

dekayi, 235

occipitomaculata, 228

Stranging, 270

Strix occidentalis caurina, 225

Survey,

Acoustic, 10

Aerial, 338

Camera, 19, 46

Electrofishing, 331
Pellet, 133, 215

Quadrat, 128, 151

Scat, 133, 215

Seismic, 120

Transect, 37, 128, 151, 228, 331

Visual, 37, 115, 120, 128, 144, 170, 203, 228, 303, 317, 338, 350, 369

Swallow, Barn, 26

Swaying, 235

Swift, Black, 144

Sylvilagus nuttalli nuttalli, 133

Syndrome, White-nose, 238

Thamnophis

ordinoides, 37

sirtalis, 228

Threlfall, M.E., 26

Thematic collection, 75, 179

Thermoregulation, Behavioural, 228

Thompson, B. Effects of Nesting Bald Eagles (Haliaeetus leucocephalus) on Behaviour and Reproductive Rates in a Great Blue Heron (Ardea herodias) Colony in Ontario, 369-371

Thymelicus lineola, 63

Tijman, B.A., 26

Trachipterus arcticus, 325

Trap

Camera, 19

Pitfall, 141

Trends

Population density, 170

Publication, 1

Temporal, 1

Treeline, 55

Trifolium resupinatum, 328

Turcotte, Y., J.-F. Lamarre, J. Bety. Annual and Seasonal Variation in Shorebird Abundance in the St. Lawrence Estuary during Fall Migration, 203214

Turtle

Leatherback, 120

Snapping, 254

Uria lomvia, 69

Ursus, 350

americanus, 225

Vanderwolf, K.J., D. Malloch, D.F. McAlpine. Psychrotolerant Microfungi Associated with Deer Mice (Peromyscus maniculatus) in a Whitenosed Syndrome Positive Bat Hibernaculum in Eastern Canada, 238-245

Variation, Colour, 335

Vegetation, Alpine, 164

Vulpes vulpes, 19 
Wagnon, C.J., T.L. Serfass. Use of Camera Traps Provides Insight into the Feeding Ecology of Red Foxes (Vulpes vulpes), 19-22

Walker, L.E., 303

Web, Food, 350

Whale

Bowhead, 270

Sperm, 120

Whitaker, A.N., K.F. Beazley. Evidence for the Historical Occurrence of Wolves (Canis spp.) in Nova Scotia, Canada, 32-36

Whitaker, D. Expanded Range Limits of Boreal Birds in the Torngat Mountains of Northern Labrador, $55-62$

Wildlife, Urban, 37

Windels, S.K., 252
Wolf

Eastern, 32

Gray, 23, 32, 215, 252

Wolves, 32

Woodland, Oak, 128

Wyoming, 303

Yukon, 221

Kluane National Park, 344

Zapus, 141

Zonitoides arboreus, 128

Zurbrigg, E., I. Brodo, J. Cipriani, C. Hanrahan, K. McLachlan Hamilton. The Ottawa Field-Naturalists' Club Awards for 2016, presented February $2017,111-114$ 


\section{Index to Book Reviews}

\section{Botany}

Beaudoin, A.B. "Ancient Pathways, Ancestral Knowledge: Ethnobotany and Ecological Wisdom of Indigenous Peoples of Northwestern North America. Volume 1: The History and Practice of Indigenous Plant Knowledge. Volume 2: The Place and Meaning of Plants in Indigenous Cultures and Worldviews" by Nancy J. Turner, 2014, 187-188

Blain, A. "Curieuses histoires de plantes du Canada. Tome 1: 1000-1670, Tome 2: 1670-1760, Tome 3: 1760-1867" by Alain Asselin, Jacques Cayouette, and Jacques Mathieu, 2014, 2015, 2107, 286-287

Brunton, D.F. "Exploring the Limestone Barrens of Newfoundland and Labrador" by M. Burzynski, H. Mann, and A. Marceau, 2017, 374

Brunton, D.F "Flora of Florida Volume IV (Dicotyledons, Combretaceae through Amaranthaceae" by R.P. Wunderlin, B.F. Hansen, and A.R. Franck, 2017, 375

Catling, P.M. "Vascular Plants of Alberta, part 1: Ferns, Fern Allies, Gymnosperms, and Monocots" by John G. Packer and A. Joyce Gould, 2017, 8081

Iles, M. "Some Useful Wild Plants: A Foraging Guide to Food and Medicine from Nature. Revised Edition" by Dan Jason, 2017, 188-189

Lamb, E. "Plant Ecology: Origins, Processes, Consequences. Second Edition" by Paul Keddy, 2017, 373-374

\section{Entomology}

Bocking, E. "The Secret Life of Flies" by Erica McAlister, 2017, 287-288

Cottam, B. "Insects: Their Natural History and Diversity: With a Photographic Guide to Insects of Eastern North America, Second Edition, Revised and Updated" by Stephen A. Marshall, 2017, 288-289

\section{Ornithology}

John, R. "Raptors: The Curious Nature of Diurnal Birds of Prey" by Keith Bildstein, 2017, 289-290

John, R. "The Australian Bird Guide" by Peter Menkhorst, Danny Rogers, Rohan Clarke, Jeff Davies, Peter Marsack, and Kim Franklin, 2017, 290291

\section{Other}

Armstrong, T. "Coexistence: The Ecology and Evolution of Tropical Biodiversity" by Jan Sapp, 2016, 295-296

Bocking, E. "The Carbon Code: How You Can Become a Climate Change Hero" by Brett Favaro, 2017, $85-86$

Brooks, R. "The Book that Changed America. How Darwin's Theory of Evolution Ignited a Nation" by Randall Fuller, 2017, 194-195

Brownstein, D. "The Sustainability Dilemma: Essays on British Columbia Forest and Environmental History" by Robert Griffin and Richard A. Rajala, 2017, 197-199

Burke, T. "The Eye of the Sandpiper: Stories from the Living World" by Brandon Keim, 2017, 296297

Cottam, B. "Protecting the Planet: Environmental Champions from Conservation to Climate Change" by Budd Titlow and Mariah Tinger, 2016, 86-87

Cottam, B. "Alexander Wilson: Enlightened Naturalist. Aperçus: Histories Texts Cultures Series" by Edward H. Burtt, Jr., 2016, 87-89

Cottam, B. New Titles, 90-93, 199-201, 297-300, 382-384

Cottam, B. "Pilgrims of the Air: The Passing of the Passenger Pigeons" by John Wilson Foster, 2014/2017, 380-381

Gaston, T. "Ice Blink: Navigating Northern Environmental History" by Stephen Bocking and Brad Martin, 2017, 86

Gaston, T. "Mourning Nature: Hope at the Heart of Ecological Loss and Grief" by Ashlee Cunsolo and Karen Landman, 2017, 192

Gaston, T. "Rise of the Necrofauna: The Science, Ethics and Risks of De-Extinction" by Britt Wray, 2017, 293

Halliday, W.D. "The Arctic Guide: Wildlife of the Far North" by Sharon Chester, 2016, 196

Smith, C.M. "Best Places to Bird in British Columbia" by Russell Cannings and Richard Cannings, 2017, 85

Smith, C.M. "This River Beneath the Sky: A Year on the Platte" by Doreen Pfost, 2017, 193

Smith, C.M. "The Magnificent Nahanni: The Struggle to Protect a Wild Place" by Gordon Nelson, 2017, 293-294

Smith, T. "Blooms. An Illustrated History of the Ornamental Gardens at Ottawa's Central Experimental Farm" by Richard Hinchcliff, 2016, 84

Tegler, B. "Drawdown: The Most Comprehensive Plan Ever Proposed to Reverse Global Warming" by Paul Hawken, 2016, 197 


\section{Poetry}

Citron, M. "Welcome to the Anthropocene" by Alice Major, 2018, 372-373

\section{Zoology}

Armstrong, T. "Encyclopedia of Whales, Dolphins and Porpoises" by Erich Hoyt, 2017, 377-379

Burke, T. "Flock Together: A Love Affair with Extinct Birds" by B.J. Hollars, 2017, 190-191

Halliday, W.D. "Marine Fishes of Arctic Canada" by Brian W. Coad and James D. Reist, 2017, 377

Lauff, R. "Fireflies, Glow-worms, and Lightning Bugs: Identification and Natural History of the Fireflies of the Eastern and Central United States and Canada” by Lynn Frierson Faust, 2017, 191-192

Lauff, R. "Great White Shark: Myth and Reality" by Alexandrine Civard-Racinais, 2017, 379

Lauff, R. "The Skeleton Revealed: An Illustrated Tour of the Vertebrates" by Steve Huskey, 2017, 380

Lennox, R.J. "Ecology of Salmonids in Estuaries around the World: Adaptations, Habitats, and Conservation" by Colin D. Levings, 2016, 81-82

Smith, C.M. "Birdmania: A Remarkable Passion for Birds" by Bernd Brunner, 2017, 189-190

Way, J. "Heart of a Lion: A Lone Cat's Walk Across America" by William Stolzenburg, 2016, 82-84

Way, J. "Wolves of the Yukon" by Bob Hayes, 2010, 291-293

Way, J. "The Wolf: A True Story of Survival and Obsession in the West" by Nate Blakeslee, 2017, 375376 